\title{
A Non-galvanic D-band MMIC-to-Waveguide Transition Using eWLB Packaging Technology
}

\author{
Ahmed Hassona ${ }^{1}$, Zhongxia Simon He ${ }^{1}$, Chiara Mariotti ${ }^{2}$, Franz Dielacher $^{2}$, Vessen Vassilev ${ }^{1}$, Yinggang Li ${ }^{3}$, \\ Joachim Oberhammer ${ }^{4}$, Herbert Zirath ${ }^{1}$ \\ ${ }^{1}$ Chalmers University of Technology, Gothenburg, Sweden \\ ${ }^{2}$ Infineon Technologies Austria AG, Villach, Austria \\ ${ }^{3}$ Ericsson AB, Gothenburg, Sweden \\ ${ }^{4}$ KTH Royal Institute of Technology, Stockholm, Sweden
}

\begin{abstract}
This paper presents a novel D-band interconnect implemented in a low-cost embedded Wafer Ball Grid Array (eWLB) commercial process. The transition is realized through a patch slot antenna directly radiating to a standard waveguide opening. The interconnect achieves low insertion loss and good bandwidth. The measured minimum Insertion Loss (IL) is $2 \mathrm{~dB}$ and the average is $3 \mathrm{~dB}$ across a bandwidth of $22 \%$ covering the frequency range 110-138 $\mathrm{GHz}$. In addition, the structure is easy to integrate as it does not require any special assembly nor any galvanic contacts. Adopting the low-cost eWLB process and standard waveguides makes the transition an attractive solution for interconnects beyond $100 \mathrm{GHz}$.
\end{abstract}

Index Terms-D-band, interconnects, waveguide transition, eWLB, millimeter waves, THz.

\section{INTRODUCTION}

The ever increasing advance in semiconductor technologies makes mm-wave technologies very attractive for several wireless applications from telecom to safety, production quality check and several other applications [1].

In this context, one of the biggest challenges that researchers face, is the realization of low-loss and low-cost interconnection and high-level integration. Various approaches are proposed in literature in order to couple the RF signal to the MMIC at mm-wave frequencies. One possibility is the integration of the antenna on chip, which is a very compact solution with the drawback of low antenna efficiency and limited bandwidth since most of the broad-side antennas are resonant structures [2].

Another option is to couple the MMIC directly to a waveguide and hence achieve efficient coupling over broader frequency range. However, most highly integrated circuits are relatively large in size with respect to the wavelength and therefore the integration of MMIC-to-waveguide transitions on chip is hardly possible. In this case, a separate transition is needed not only because of the area limitation but also to prevent waveguide modes from leaking into the circuit cavity. The drawback of this solution is that it requires a bond-wire interface between the waveguide-transition and the MMIC. The use of bondwires is not suitable at such high frequencies since they introduce inductive effects and require special measures to provide reasonable return-loss for the interface [2].

Embedded wafer level ball grid array (eWLB) provides an attractive solution for packaging MMIC into a ball grid array surface mountable module with hundreds of I/O connections. Low frequency I/O has been proposed and studied in [3] using standard eWLB connections. High frequency I/O connection up to $100 \mathrm{GHz}$ has been proposed using antenna-like structures [3]. To the authors' knowledge, sub mm-wave interconnects on eWLB technology has not been proposed before.

In this work, a generic approach for a MMIC-to-waveguide transition based on eWLB process is proposed with the support of experimental results. The choice of eWLB technology is motivated by the need of a low-cost and highvolume process for interconnects that operate at $\mathrm{mm}$-wave frequencies. This has been a major challenge hindering commercialization of mm-wave systems. To the authors' knowledge, this is the first attempt to fabricate a transition beyond $100 \mathrm{GHz}$ using eWLB process.

\section{TRANSITION DESIGN}

The proposed solution consists of an eWLB chip with an embedded MMIC, Ball grid array (BGA) for low frequency $\mathrm{I} / \mathrm{Os}$, eWLB antenna for I/Os above $100 \mathrm{GHz}$, a PCB to support eWLB and a standard air-filled metal waveguide. The complete solution is shown in Fig. 1. The eWLB process provides two metal layers named redistribution layers (RDL), mainly used for $\mathrm{I} / \mathrm{O}$ connections. In this work, the redistribution layers are also used to realize the antenna that couples the signal into the waveguide avoiding galvanic connection. The dielectric constant of the substrate is 3.2 which is suitable for limiting the leakage into the substrate. The substrate height is $0.45 \mathrm{~mm}$ which is close to quarter wavelength at D-band and hence, placing the chip on a conductive surface, would make it act as a backshort leading to better radiation into the waveguide.

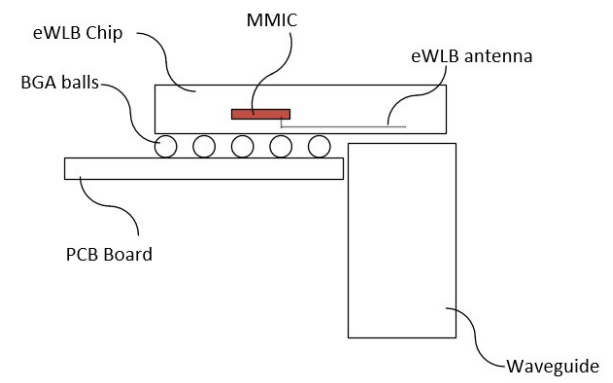

Fig. 1. Simplified schematic for the proposed solution 
The patch slot antenna is implemented on the top redistribution layer. The antenna's slot width is $0.15 \mathrm{~mm}$. The antenna faces the waveguide opening which is mounted perpendicular to the eWLB level. The antenna feed lines are implemented as coplanar waveguide (CPW) lines. The ground lines are implemented on the top redistribution layer and the signal feed line is implemented on the bottom redistribution layer to avoid shorting the signal to ground in case the waveguide walls made contact with the eWLB's surface. The CPW lines can be used to connect the transition to any MMIC.
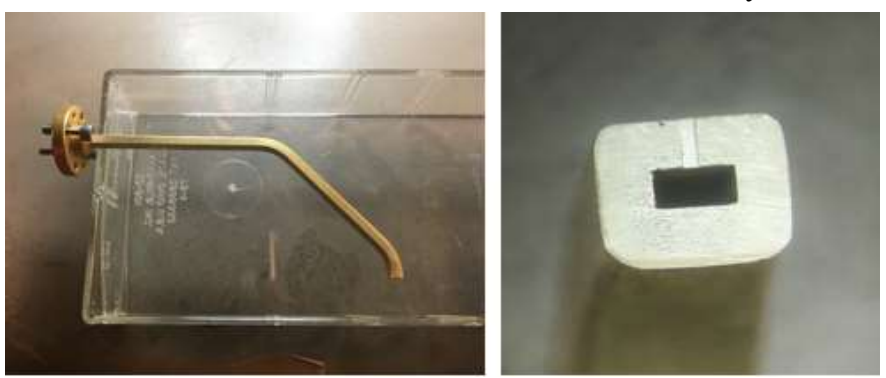

Fig. 2. Waveguide's $90^{\circ}$ bend with one end connected using a flange and the other end facing the eWLB

A commercial D-band waveguide $90^{\circ}$ bend was used to test this design. A 30-um-depth slot was machined in the waveguide wall above the feeding lines as shown in Fig. 2 in order to avoid disturbing the CPW mode.

The sides of the chip were metallized by machining a channel through a metal base with the same height as the chip. Metalizing the side of chip resulted in an improvement of 0.5 $\mathrm{dB}$ in insertion loss.

Fig. 3 shows a photo of the fabricated prototype. The chip is implemented as a back-to-back solution allowing straightforward testing and calibration. Alignment markers were used on-chip to correctly align waveguide openings to the antenna to achieve maximum coupling. CPW line structures were also added on chip to calibrate line losses and extract the transition loss accurately.

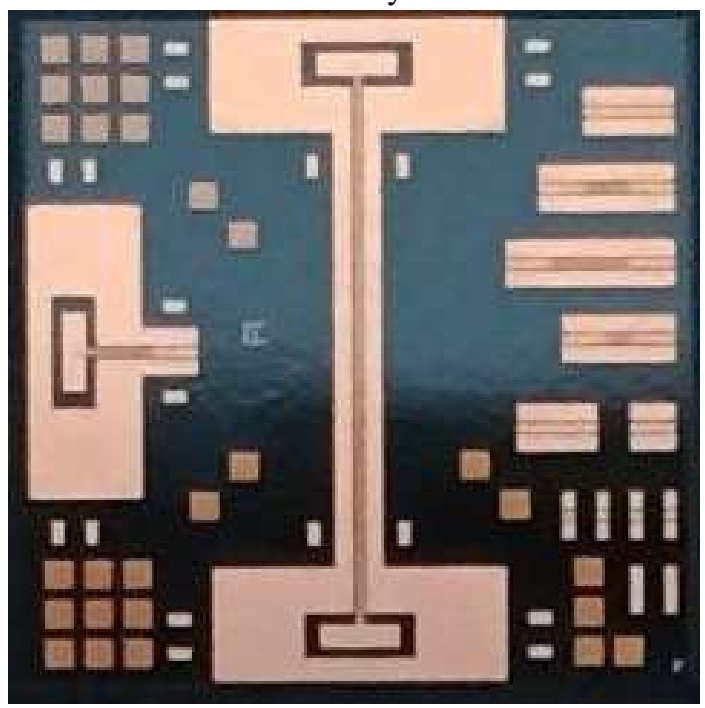

Fig. 3. Chip photo

\section{EXPERIMENTAL RESULTS AND DISCUSSION}

Measurements were performed in a back-to-back fashion. The waveguide bends were mounted on a probe station in order to provide accurate alignment of the waveguide openings. The measurement structure is based on a PNA-X network analyzer up to $67 \mathrm{GHz}$, a pair of frequency extension modules to up/down-convert the signal frequency to D-band The measurement setup is shown in Fig. 4. Two-port calibration was performed till the inputs of the waveguide bends.

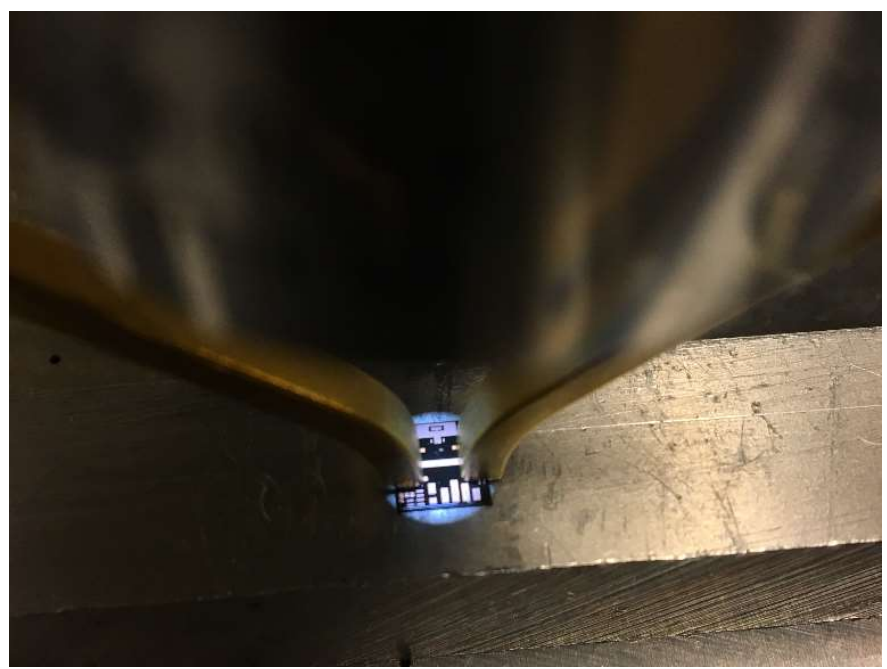

Fig. 4. Measurement setup

The losses of the bends were measured separately and deembedded manually. Measurements show a loss of $0.9 \mathrm{~dB}$ per bend at D-band. De-embedded measurement results showed an average insertion loss of only $3 \mathrm{~dB}$ per transition as reported in Fig. 5. The minimum achieved insertion loss is 2 $\mathrm{dB}$. The transition shows a relatively wide bandwidth of $22 \%$ covering the range 110 to $138 \mathrm{GHz}$. Simulations have been performed using HFSS 3D EM simulator and compared to

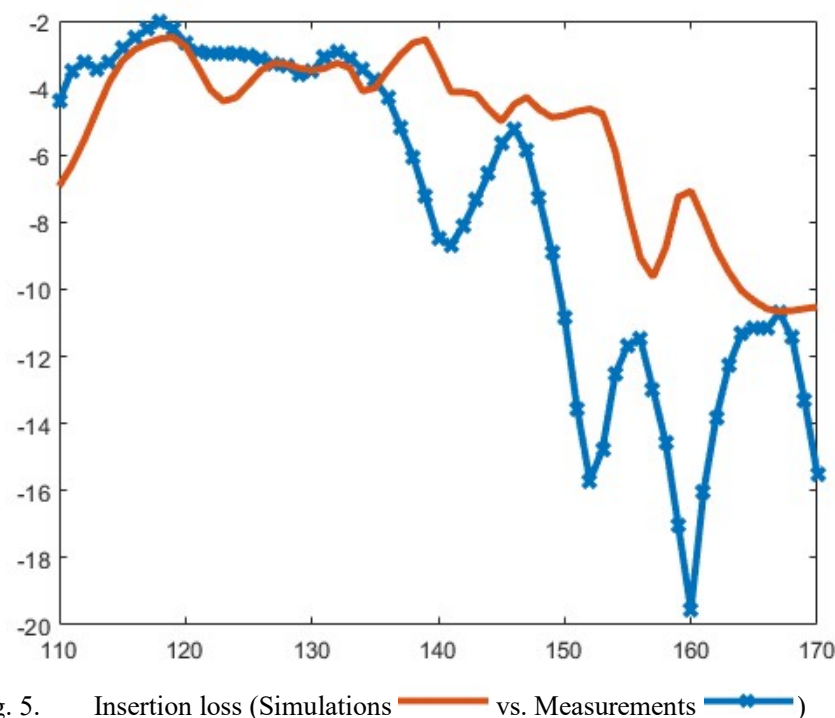


TABLE I

COMPARISON OF DIFFERENT MMIC-TO-WAVEGUIDE TRANSITIONS

\begin{tabular}{|c|c|c|c|c|c|}
\hline Ref. & Description & $\begin{array}{c}\text { Frequency } \\
(\mathbf{G H z})\end{array}$ & $\begin{array}{c}\text { Insertion loss } \\
\mathbf{( d B )}\end{array}$ & Bandwidth & Technology \\
\hline$[4]$ & $\begin{array}{c}\text { Micromachined Sub-THz } \\
\text { Interconnect Channels }\end{array}$ & $151-160$ & 9 & $5.7 \%$ & Micromachined Si \\
\hline$[5]$ & Dielectric waveguide & $146-198$ & 4.9 & $30 \%$ & $\mathrm{HR} \mathrm{Si}$ \\
\hline$[6]$ & Dielectric waveguide & $140-156$ & 7 & $10.8 \%$ & PMMA dielectric waveguide \\
\hline This work & $\begin{array}{c}\text { Non-galvanic antenna } \\
\text { based transition }\end{array}$ & $110-138$ & 3 & $22 \%$ & eWLB \\
\hline
\end{tabular}
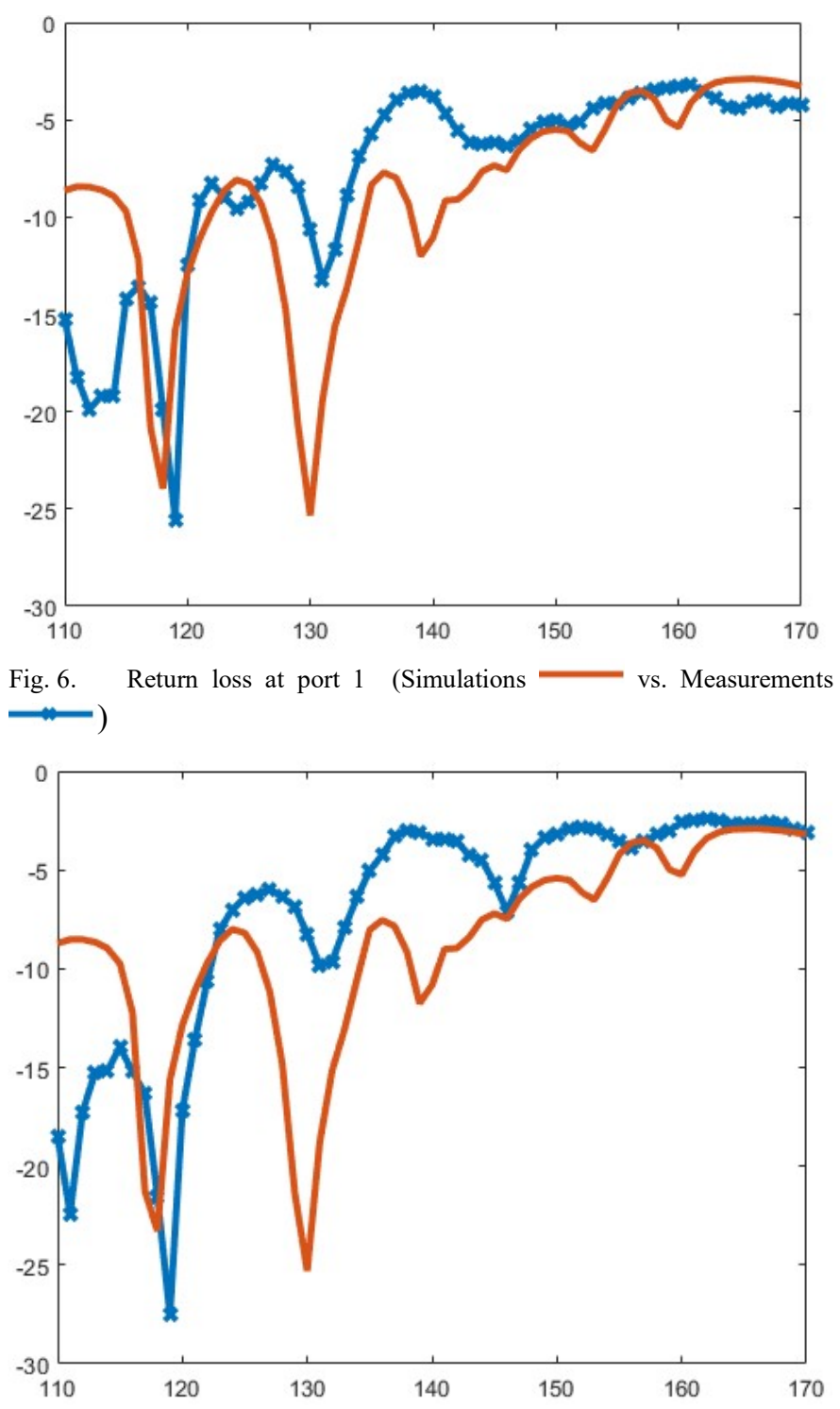

Fig. 7. Return loss at port 2 (Simulations - vs. Measurements measurements. It is noteworthy that the measured frequency response shows a shift towards lower frequencies compared to simulated response. The return losses at both ends are shown in Fig. 6 and Fig. 7. Simulations show good agreement with measurements especially for nulls locations. The performance of the proposed transition is compared to similar work at the same frequency in Table 1 . The comparison shows that the proposed transition has the lowest insertion loss and a relatively wide bandwidth.

\section{CONCLUSION}

A novel D-band MMIC-to-waveguide transition on a commercial low cost eWLB process has been presented. The transition is implemented using a patch slot antenna radiating to a standard waveguide. Experimental results demonstrate that the transition achieves low insertion loss of $3 \mathrm{~dB}$ and a bandwidth of $22 \%$ at D-band. The transition does not require galvanic contacts and forms a generic interconnect that can be integrated with any MMIC/PCB. The proposed transition represents a solid step towards high volume commercialization of $\mathrm{mm}$-wave and $\mathrm{THz}$ systems as it is implemented in a well-established commercial technology.

\section{Acknowledgment}

This project has received funding from the European Union's Horizon 2020 research and innovation program under grant agreement No. 644039.

\section{REFERENCES}

[1] S. Carpenter, M. Abbasi, and H. Zirath, "Fully integrated D-band direct carrier quadrature $(\mathrm{I} / \mathrm{Q})$ modulator and demodulator circuits in $\operatorname{InP}$ DHBT technology," IEEE Trans. Microw. Theory Tech., vol. 63, no. 5, pp. 1666-1675, May 2015.

[2] V. Vassilev et al., "Integrated front-ends up to $200 \mathrm{GHz}, 2011$ IEEE MTT-S International Microwave Workshop Series on Millimeter Wave Integration Technologies, Sitges, 2011, pp. 57-60.

[3] M. Wojnowski et al., "Embedded Wafer Level Ball Grid Array (eWLB) Technology for Millimeter-Wave Applications", ECTC 2011

[4] Bo Yu, Yuhao Liu, Xing Hu, Xiaoxin Ren, Xiaoguang Liu and Qun Jane $\mathrm{Gu}$, "Micromachined sub- $\mathrm{THz}$ interconnect channels for planar 
silicon processes," 2014 IEEE MTT-S International Microwave Symposium (IMS2014), Tampa, FL, 2014, pp. 1-3.

[5] B. Yu, Y. Liu, Y. Ye, X. Liu and Q. J. Gu, "Low-loss and Broadband GBand Dielectric Interconnect for Chip-to-Chip Communication," in IEEE Microwave and Wireless Components Letters, vol. 26, no. 7, pp. 478-480, July 2016.

[6] M. Hitzler, S. Saulig, L. Boehm, W. Mayer and C. Waldschmidt, "MMIC-to-waveguide transition at $160 \mathrm{GHz}$ with galvanic isolation," 2016 IEEE MTT-S International Microwave Symposium (IMS), San Francisco, CA, 2016, pp. 1-4. 